\title{
Las fronteras de la medicalización: tensiones en torno a la identificación y valoración de la desnutrición infantil en un centro de atención primaria de la ciudad de Buenos Aires
}

\author{
The frontiers of medicalization: tensions surrounding \\ the identification and appreciation of child malnutrition \\ in a primary healthcare center of the city of Buenos Aires
}

Damián Herkovits ${ }^{1}$

\begin{abstract}
The medicalization of life and its implications for the production of subjectivities are phenomena that have been highlighted by the human sciences in the study of health and disease. Nevertheless, the analysis of its local expressions has been insufficiently covered. The scope of this paper is to explore this field by an ethnographical study of the medicalization process of child malnutrition in a primary healthcare center of the city of Buenos Aires. We will describe analytically the singularities involved in the body perception and the alimentary context by health professionals and their patients. We emphasize that the criteria of perception and moral values that encourage social positions of health professionals and recipients of their actions precluded the institutionalization of a medical vision. We conclude that the process analyzed highlights the need to exceed the medicalization approaches dealing exclusively from the angle of imposition. The social history of the groups involved and ways of establishing relationships in local settings, are essential to understand the peculiarities of these processes.
\end{abstract}

Key words Nutrition, Body, Child malnutrition, Conflict, Medicalization
${ }^{1}$ Fundación Prosama Paysandú 752-760. Ciudad Autónoma de Buenos Aires. damherk@gmail.com
Resumo La medicalización de la vida y sus implicancias en cuanto a la producción de subjetividades han sido fenómenos destacados por las ciencias humanas en el estudio de la salud y la enfermedad. Sin embargo, el análisis de sus expresiones locales permanece insuficientemente tratado. El presente trabajo tiene como propósito avanzar en esta dirección mediante el estudio etnográfico del proceso de medicalización de la desnutrición infantil en un centro sanitario de la ciudad de Buenos Aires. Abordaremos las singularidades involucradas en la percepción valorativa del cuerpo $y$ su contexto alimentario por parte de los profesionales de la salud y los destinatarios de sus acciones terapéuticas. Destacamos que los criterios de percepción y los valores morales que animan las posiciones sociales de los profesionales del sector salud y los destinatarios de sus acciones imposibilitaron la institucionalización de la visión médica. Concluimos que el proceso analizado subraya la necesidad de exceder los enfoques que abordan la medicalización exclusivamente desde el ángulo de la imposición. La historia social de los grupos involucrados y los modos de relacionamiento que establecen en escenarios locales constituyen elementos esenciales para comprender las singularidades de estos procesos.

Palabras clave Alimentación, Cuerpo, Desnutrición infantil, Conflicto, Medicalización 


\section{Introducción}

Entre los aspectos resaltados por las ciencias sociales en el análisis de los procesos de salud, enfermedad y atención, la medicalización de la vida se destaca como uno de los movimientos con mayores implicaciones en el terreno de la política, la economía y la cultura. Las modalidades en que la medicina participa de las políticas de designación de la desviación ${ }^{1,2}$, los principios y modalidades que intervienen en la construcción de sus objetos de intervención ${ }^{3-5}$ y los dispositivos terapéuticos y sanitarios destinados a la producción de subjetividades $^{6,7}$, constituyen campos de indagación fundamentales para el análisis de aquello que, de acuerdo con la tradición foucoulteana, compone la gobernabilidad de la vida en las sociedades contemporáneas. Sin embargo, las expresiones que singularizan las modalidades en que se practica la medicalización en el escenario de la vida cotidiana; las eventuales respuestas de los conjuntos sociales involucrados en este movimiento; sus potenciales efectos sobre la construcción de los espacios terapéuticos; y su consecuente impacto en la producción de subjetividades, constituyen un terreno insuficientemente explorado y de vital importancia para la comprensión de dicho fenómeno. El presente trabajo tiene como propósito adentrarnos en esta problemática mediante el análisis del proceso del tratamiento de la desnutrición infantil producido en un centro de atención primaria de la salud de la ciudad de Buenos Aires. Específicamente presentaremos los elementos fundamentales a partir de los cuales se conformó la percepción valorativa del cuerpo de los niños y su contexto alimentario entre los profesionales del sector salud y quienes conformaban su entorno doméstico. Además, abordaremos las consecuencias que tuvieron estas construcciones en el desarrollo del programa asistencial. Sostendremos que a pesar del protagonismo asignado al saber y las prácticas del sector salud, los destinatarios de sus acciones desarrollaron visiones antagónicas de la realidad somática y alimentaria de los niños. Este fenómeno, expresión local de las trayectorias y posiciones sociales de sus protagonistas, hizo de los dispositivos clínicos un campo agonístico cuyos resultados se encontraron lejos de lo esperado por los profesionales de la salud. Concluimos que el proceso analizado subraya la necesidad de exceder los enfoques que abordan la medicalización exclusivamente desde el ángulo de la imposición. La historia social de los actores que protagonizan estos procesos es un elemento ineludible para comprender los matices que en definitiva configuran las múltiples realidades del presente.

\section{La producción de la evidencia: notas sobre los recursos metodológicos desplegados}

La información que sustenta el presente trabajo fue producida en el marco de una investigación sobre alimentación, pobreza y medicalización de la vida, desarrollada entre diciembre de 2002 y septiembre de 2007 en un barrio cadenciado de la ciudad de Buenos Aires. El "barrio", definido localmente como una "villa" o "villa de emergencia", se extiende unos $2,5 \mathrm{~km}^{2}$, y cuenta con una población de 18.000 personas. La mayoría de sus habitantes reside en pequeñas casas de dos o tres habitaciones, generalmente de ladrillos sin revocar. Sin embargo, también es posible observar sectores en los que prevalecen viviendas de chapa, o incluso hechas de un conjunto variado de materiales entre los que se reconoce el cartón, la madera, el plástico y la lona. Casi la totalidad de las casas cuenta con servicios de agua, electricidad y gas envasado en garrafas. En el interior del barrio se encuentra un centro de salud con más de treinta profesionales abocados a los servicios de clínica médica, pediatría, ginecología, psicología, psicopedagogía, traumatología, nutrición, ginecología y enfermería. También existe una escuela primaria dependiente del gobierno municipal y negocios destinados a la proveeduría de artículos básicos.

El trabajo de campo se desarrolló fundamentalmente en el efector de salud y en 15 de los hogares en situación de pobreza y pobreza extrema vinculados con esta institución a partir del diagnóstico de "desnutrición" de uno de sus integrantes de entre 1 y 5 años: 8 con indicadores de "desnutrición crónica" y 7 de "desnutrición aguda". Los puntos de corte utilizados para la identificación de estos trastornos fueron talla / edad <-2 desvíos estándar para la desnutrición crónica, y peso / talla <-2 desvíos estándar para la desnutrición aguda. Los diagnósticos de todos los niños tuvieron además una confirmación clínica relacionada con la disposición de micronutrientes, el retardo en la velocidad de crecimiento o la semiología corporal ${ }^{8}$. El efector de salud fue seleccionado en primera instancia por ser, de acuerdo a los informes oficiales, uno de los que había identificado más casos de desnutrición en el conjunto de la ciudad. Por otro lado, los "casos" de "desnutrición" se seleccionaron a partir de una autoadscripción voluntaria. Para esto se solicitó a los profesionales de la salud que ofrecieran a 
los padres y cuidadores de los niños la posibilidad de mantener una reunión con el investigador luego de haber conocido el diagnóstico. A quienes aceptaron se les informó sobre los objetivos de la investigación, los procedimientos que serían empleados, así como el tratamiento, destino y confidencialidad de la información. Se explicitó además que podían retirarse total o parcialmente del estudio en el momento en que lo desearan y que esta eventualidad no sería conocida por las y los profesionales de la salud o por quienes fuesen responsables de programas o proyectos sociales con los que pudiesen estar eventualmente relacionados.

A lo largo del estudio, el enfoque metodológico adoptado fue fundamentalmente cualitativo y más específicamente etnográfico, es decir que se procuró acceder a las prácticas y sentidos de los actores en el escenario de la vida cotidiana, así como a las perspectivas y modalidades que singularizan su construcción del mundo ${ }^{9,10}$. Para esto, se realizaron observaciones con un variado grado de participación en el efector de salud y en el espacio doméstico de los hogares. En el caso del centro de salud se frecuentaron los ámbitos vinculados con las actividades de diagnóstico y el tratamiento de la desnutrición infantil. Específicamente, se asistió a las reuniones de trabajo y los dispositivos terapéuticos implementados, fundamentalmente los espacios de educación y seguimiento alimentario de los niños y sus familias. Por otro lado, en los hogares se realizaron visitas reiteradas en las que se observó con participación los ámbitos y modalidades involucrados en la obtención, preparación y utilización de los alimentos.

Cada una de las jornadas de trabajo fue textualizada en un registro exhaustivo de los relatos, acontecimientos, protagonistas y contextos que tuvieran una relación directa o indirecta con el tema de investigación. A partir de este corpus documental se identificaron diferentes aspectos vinculados con la alimentación el cuerpo y el cuidado de los niños en general y al alimentario en particular. Este trabajo fue acompañado por una serie de descripciones analíticas en las que se explicitaron líneas de interpretación teórica, vinculaciones entre los temas y principios de estructuración sobre los que se podía componer la presentación de los resultados ${ }^{11-15}$. En este sentido, el trabajo que presentamos es el fruto de un proceso de vinculación e interpretación teórico metodológica de la experiencia de campo.

\section{La construcción del cuerpo infantil y su contexto alimentario como realidad clínica: formalización perceptiva y culturalismo etiológico}

Hacia fines de diciembre de 2001, en la Argentina se desencadenaba una grave crisis social, política y económica. La ausencia de financiamiento para un déficit presupuestario crónico en las cuentas públicas, la fuga de capitales, la inmovilización de los depósitos bancarios, el aumento abrupto del desempleo, de precios y una deslegitimación de las organizaciones políticas y partidarias como vehículos de representación y acción colectiva, eran algunos de los elementos que confluyeron para conformar, en la primera mitad de 2002, uno de los escenarios más sombríos de su historia. En el plano estrictamente material y como consecuencia de este proceso, hacia mediados de 2002 el 57\% de los habitantes del país se ubicó por debajo de la línea de la pobreza, y más un cuarto por debajo de la de indigencia ${ }^{16}$.

Aunque el estado nutricional de la población no era del todo conocido, el impacto de esta conyuntura comenzó a ganar espacio en la agenda pública y social. Consecuentemente, desde agencias gubernamentales y no gubernamentales se incentivaron y fortalecieron diversas acciones tendientes a mejorar el acceso a los alimentos y los servicios de salud a los sectores más vulnerables de la población. Específicamente en la ciudad de Buenos Aires, la preocupación alimentaria impulsó la formalización de un programa destinado a la prevención y el tratamiento de trastornos ligados al déficit nutricional. Sus objetivos fundamentales eran la evaluación del crecimiento y desarrollo de los niños, el abordaje de las anomalías observadas en los procesos alimentarios y la articulación de sus hogares con instituciones y recursos destinados a mejorar el acceso a los alimentos ${ }^{17}$. La implementación de las actividades del programa, desarrolladas en los 40 centros de atención primaria dependientes del gobierno de la ciudad de Buenos Aires, conformó un marco institucional y un incentivo a la sensibilidad de muchos de los profesionales de la salud con relación al problema alimentario. Entre sus expresiones prácticas se destacaron la evaluación del estado nutricional de las y los niños, el contacto eventual de las familias con diversas fuentes de abastecimiento y el desarrollo de actividades de promoción de hábitos alimentarios saludables. Si bien muchas de las actividades mencionadas preexistían al programa, este proceso las extendió y fortaleció. De esta manera se 
fomentó la introducción del cuerpo de los niños y sus contextos alimentarios como un objeto de intervención sanitaria cuyas tensiones se expresaron en la articulación de dos elementos fundamentales: la percepción del cuerpo y la etiología proyectada sobre el padecimiento.

Las dimensiones y proporciones corporales, su contextura y consistencia, son aprendidas a partir de modalidades de percepción y formas de clasificación histórica y culturalmente sitiadas. En este sentido, la mirada valorativa sobre su volumen peso y estatura, pero también sobre sus formas, maneras y posición, es producto de un escenario de disputas en el que participan diversos grupos sociales ${ }^{18}$. En el campo de la salud nutricional, la percepción valorativa de las dimensiones y proporciones corporales se haya enraizada en una intención clasificatoria que persigue asentar una distinción exhaustiva y excluyente entre lo normal y lo patológico. Esta taxonomía es construida fundamentalmente a partir de las relaciones que se establecen entre las medidas antropométricas. La comparación del peso, la talla y la edad de quien es evaluado con un valor poblacional de referencia permite clasificar su cuerpo dentro de estos dos conjuntos fundamentales. Los profesionales de la salud actualizaron esta perspectiva en su observación del crecimiento y desarrollo de los niños. De esta manera distinguieron las modalidades de malnutrición al margen de la subjetividad del observador circunstancial. Por otro lado, la especificidad de la acción médica se centró en la creación de diversos dispositivos de educación alimentaria. Allí se pretendió tratar la etiología de los casos, postulada como el conjunto de creencias y prácticas propias de los contextos alimentarios domésticos que operaban deletereamente en la producción del estado nutricional de los niños. Fundamentalmente se abordaron las modalidades en que los alimentos eran preparados, cocidos y distribuidos, así como los vínculos que los niños mantenían con esta actividad cotidiana. Finalmente, las acciones del programa se completaron con políticas destinadas a mejorar el acceso a los alimentos en los hogares: específicamente se distribuyeron mensualmente una caja de alimentos básicos por niño diagnosticado con desnutrición y con el acceso a comedores sociales y comunitarios de la zona.

Más allá de las prestaciones alimentarias -a cargo de organismos no vinculados estrictamente con el sector salud- la clasificación de los cuerpos y el tipo de intervención evidencian rasgos característicos de la forma en que se construye la perspectiva clínica de la realidad. La formalización de la percepción proyectada sobre la morfología de los niños hizo que los juicios de los profesionales se afirmaran sobre principios epistemológicos del positivismo. Esta perspectiva, que constituye el sentido común del saber médico, permitió la universalización de los diagnósticos y consecuentemente una identificación común de los casos a intervenir. Por otro, en la etiología proyectada primó una perspectiva culturalista enraizada no sólo en la tradición epistemológica de los profesionales de la salud, sino además en la posición social y los valores que vertebran su profesión. Entre los aspectos claves que conformaban la causalidad de la malnutrición, los saberes de padres madres y cuidadores -definidos en términos de creencias, mitos y tradiciones- resultaron factores claves para comprender el estado nutricional de los niños. La implementación de dispositivos educativos persiguió consecuentemente la modificación de estos saberes por otros asentados en el conocimiento "científico" y opuestamente benéficos para su realidad somática. Esta práctica, que supone en los destinatarios de las intervenciones sanitarias la existencia de una racionalidad universal, susceptible de recibir, evaluar e incorporar a la vida cotidiana las prescripciones impartidas, redujo la etiología de la malnutrición a su componente cognitivo. Si bien en el plano discursivo los profesionales de la salud destacaban dimensiones socioeconómicas como la "pobreza", o la "exclusión" entre los factores fundamentales para comprender el problema, dicha focalización constituyó una operación necesaria para adecuar la construcción del objeto clínico a su posición profesional. Entre los trabajadores del sector salud la disponibilidad y el ejercicio de un saber conforman un elemento constitutivo de la "profesionalidad" de sus acciones. Sin embargo, el impulso por modificar aspectos que se definen como indeseables resultó definitorio. La acción sobre la realidad adquiere el carácter de un valor central en la posición social del sector terapéutico. Su ejercicio se efectiviza más allá de los saberes que componen su competencia específica o la capacidad de resolución que puedan exhibir sobre los problemas identificados. Ningún profesional de la salud puede resolver la falta de alimentos en los hogares desde su consultorio. Sin embargo, determinados aspectos relacionados con la modalidad en que son consumidos, o las formas de cuidado y alimentación de los niños, permiten una intervención por medio de los recursos más habituales: la asistencia clínica y las actividades educativas como instancias preventivas y reparadoras. Esta construcción del 
objeto clínico permitió el desarrollo de prácticas sustentadas en los valores que constituyen su identidad profesional y que legitiman consecuentemente su posición en el campo social y sanitario.

La percepción formalizada del cuerpo, la clasificación exhaustiva y universal entre lo "normal" y lo "patológico" y el horizonte "culturalista" de la etiología del padecimiento, constituyen elementos característicos de la forma en que se construyen los objetos terapéuticos en el campo de la intervención sanitaria. Sin embargo, y a pesar de su carácter hegemónico, al cabo de tres años de implementación, los resultados a nivel local arrojados por el programa resultaron insatisfactorios para los profesionales que lo ejecutaban. La cantidad de "altas" correspondía a menos del 10\% del total de ingresados y la deserción, es decir, el abandono de los "pacientes" antes de recuperar la condición de salud según los parámetros normatizados, superaba el 50\% del total de los diagnosticados. Además, de acuerdo con la valoración de los profesionales, la respuesta de sus "pacientes" a las indicaciones pendulaba entre la indiferencia y la distancia. Con la pobreza como trasfondo ineludible, muchos de los profesionales calificaban a los hogares como "disfuncionales", entornos donde nuevamente lo "cultural” o las "características psicológicas" de sus integrantes protagonizaban las explicaciones en torno a la "falta de conciencia" sobre el problema nutricional de los niños. ¿Cuáles eran las características de los espacios domésticos sobre las que se inscribió esta representación? ¿Qué singularidades presentaban los horizontes socioculturales desde los cuales resultaba posible? Como veremos a continuación, la respuesta a estos interrogantes se inscribió en las trayectorias en las que se anclaba la misma posibilidad de supervivencia material de los grupos, cierta naturalización de la escasez extrema que sustentó formas antagónicas de percibir y valorar tanto el cuerpo como su realidad alimentaria.

\section{El entorno de los “desnutridos" frente al diagnóstico: continuidad perceptiva y normalización etiológica}

Aunque no modificó sustancialmente sus modos de vida, el período 2001-2002 representó para los destinatarios de las acciones sanitarias un agravamiento de las condiciones y posibilidades materiales que componían su contexto cotidiano. Marginados de la sociedad laboral desde hacía años, incorporados a los discontinuos, focalizados y magros programas sociales sosteni- dos por organizaciones gubernamentales y no gubernamentales, la "crisis" se expresó fundamentalmente como un endurecimiento del mercado de trabajo informal en el que estaban eventualmente insertos. Este fenómeno fue acompañado por un incremento en el número de comedores existentes en el barrio, pero además por un aumento de la circulación de bienes y servicios entre conocidos y parientes. Con relación a la conformación de los platos cotidianos, los interlocutores manifestaron una mayor presencia de preparaciones hervidas, abundantes en ingredientes baratos como el arroz o las pastas pero escasos en verduras y carnes, de mayor precio en el mercado.

Tanto la extensión e intensificación de las redes como la recurrencia de las preparaciones hervidas no representaron novedades cualitativas con relación a las prácticas que conformaban la habitualidad doméstica. En realidad, sólo expresaron variaciones de una misma racionalidad que permitía y permitió construir un repertorio culinario a bajo costo ${ }^{19-21}$. En este sentido, se trató de una profundización de los habitus que, en el sentido que Bourdieu le otorga al término ${ }^{22,23}$, habían posibilitado la alimentación de los grupos en el pasado. Aún si sus deficiencias podían ser reconocidas por sus consumidores, estas preparaciones continuaron siendo, sólo que con más énfasis, la única posibilidad de producir la saciedad cotidiana.

La creciente preocupación estatal por tutelar el cuerpo y la alimentación de los niños operó en este contexto como una práctica disruptiva de la cotidianeidad. Entre quienes se habían incorporado al programa a partir de un diagnostico nutricional deficitario, la construcción clínica de la realidad somática y alimentaria de los niños no sólo resultó ser una visión especializada y ajena sobre el cuerpo infantil, sino además, en alguna medida, rebatible. Este fenómeno puede resultar llamativo si tenemos en cuenta la legitimación y la extensión de la visión médica sobre el cuerpo en general y la salud y la enfermedad en particular. Más aún si consideramos que la accesibilidad al sistema de salud y su universo de representaciones se encuentra geográfica y temporalmente extendido al conjunto de los casos abordados. Sin embargo, para madres, padres y cuidadores, la distancia entre el lenguaje profesional y la propia percepción y evaluación de su cuerpo fue un juicio extendido tanto en el diagnóstico como en la etiología de los casos.

Con respecto al diagnóstico, si desde la mirada médica la morfología de los niños con desnu- 
trición presentaba algún tipo de anomalía, desde la perspectiva de las madres y cuidadores, éstos no diferían de quienes eran diagnosticados como "normales": "yo lo veo bien, es igual a sus hermanos o los primitos, por ahí un poco más flaco, nada más" (Marta, 32 años, madre de un niño de 3 años con indicadores de desnutrición crónica); "no sé, yo no creo que esté mal, o si él está mal, están mal todos los chicos del barrio" (Gabriela, 27 años, madre de una niña de 4 con indicadores de desnutrición crónica). Además, la delgadez o pequeñez de sus cuerpos, expresiones características de la desnutrición crónica o aguda, era homologada a la herencia biológica de los padres o familiares cercanos, subrayando así cierta continuidad doméstica: "yo también siempre fui muy flaca, mi mamá me decía que cuando era chica parecía un palo de escoba y el padre es flaco también, así que gordo no iba a salir" (Georgina de 24 años, madre de un niño de 4 años con indicadores de desnutrición aguda); "Samuel es chiquitito, pero como mi papá, él también era así, el pobre salió como el abuelo, no tuvo suerte" (Lorena, 37 años, madre de un niño de 5 años con indicadores de desnutrición crónica).

En segundo lugar, un elemento fundamental que reforzó la exclusión de las y los niños del terreno de lo patológico fue la evaluación de su comportamiento. El padecimiento suele ser inmediatamente asociado con la experiencia del sufrimiento o la incapacidad de desempeñarse en concordancia con las expectativas habituales ${ }^{24-}$ ${ }^{26}$. Sin embargo, de acuerdo a las madres y cuidadores, tanto los "flaquitos" como los "bajitos" se desenvolvían cotidianamente sin impedimento alguno. No sólo su comportamiento era semejante al recordado en su propia historia personal, sino que también les parecía similar al observado en sus otros hijos, o en los hijos de sus vecinas y familiares: "Micaela está flaca, se le ven los huesos, es menudita, pero está bien, juega, corre". (Jorgelina, 25 años, madre de una niña de 4 años con indicadores de desnutrición aguda). "Yo creo que Sabrina a lo sumo se enferma a veces, pero qué va a estar mal si tengo que estar corriéndola todo el día" (Marta, 28 años, madre de una niña de 4 años con indicadores de desnutrición crónica).

Finalmente, un tercer elemento se relacionó con la observación del crecimiento y desarrollo de los niños. Más allá de las apreciaciones que pueden realizarse desde el sector salud, las madres y cuidadores observaban un hecho que les parecía incuestionable: los niños efectivamente eran más grandes año tras año: "Manuel crece, por más que le digan que está desnutrido, yo veo que juega, que crece, que está bien [...] vos te das cuenta porque la ropita que tienen al tiempo ya le queda chica, no le sirve más..." (Marina, 31 años, madre de un niño de 4 años con indicadores de desnutrición crónica). "A pesar de que está flaco y todo lo que dicen, él crece, es sanito, no tiene problemas" (Vanina, 29 años, madre de un niño de 3 años con indicadores de desnutrición aguda). Si bien las carencias alimentarias pueden detener el desarrollo de la talla de los niños en términos de su potencialidad, la percepción del retraso puede requerir una visión especializada que permita captar las sutilezas de este proceso. Entre los integrantes de los grupos domésticos a los que estos pertenecen, dicha visión no compuso la perspectiva natural a partir de la cual se producía la vida cotidiana.

Por otro lado, si la confluencia entre estos factores contribuyó a generar cierta distancia entre el diagnóstico profesional y el de quienes conformaban el contexto inmediato de los niños, el horizonte cultural vinculado con la construcción de la maternidad y los atributos de su identidad legítima contribuyeron a socavar su etiología. De acuerdo con el orden moral que conforman los grupos domésticos, el cuidado y la alimentación de los niños son funciones asignadas a las mujeres en general y a las madres en particular. Consecuentemente, la etiología del padecimiento representó en alguna medida un cuestionamiento a su idoneidad en el desempeño de este rol. Desde la perspectiva de las madres, la “desnutrición” las hacía protagonistas de cierta condena moral que teñía el entorno en el que el cuerpo de los niños era producido: "a mí me pone mal cuando piensan que en la casa no come, o que yo no me ocupo de él” (Marina, 31 años, madre de un niño de 4 años con indicadores de desnutrición aguda); "y que te digan que el chico no come bien te jode, porque además aunque uno diga lo que diga, siempre parece que estás ocultando algo, que hay algo que no está bien y que vos querés zafar" (Marta, 25 años, madre de una niña de 4 años con indicadores de desnutrición crónica). Frente a esta situación, sus respuestas compusieron un ejercicio de normalización de la situación, sustentado en tres elementos recurrentes. En primer lugar, podían destacar los atributos básicos que definen su concepción del rol, especialmente las prácticas destinadas al cuidado de los niños: "puede ser que yo no coma viste, que yo me vaya a la cama sin comer, pero los chicos en casa nunca se van a la cama con la panza vacía” (Analía, 29 años, madre de un niño 
de 2 años con indicadores de desnutrición crónica). "Acá siempre hacemos todo por los chicos, los cuidamos lo mejor que podemos y a la hora de la comida de una u otra forma hacemos que ellos tengan, que puedan comer algo, aunque los grandes no tengamos nada" (María Elena, 42 años, madre de un niño de 5 , con indicadores de desnutrición aguda). Esta reafirmación discursiva del cuidado, subrayada por prácticas asociadas con la abnegación como en el caso de Analía, destacan un horizonte cultural que aleja a la anormalidad como un resultado posible: los niños no pueden estar mal porque sus madres y cuidadores hacen todo lo que está a su alcance por ellos, incluso al punto del sacrificio de la alimentación personal. Además, un segundo elemento se relacionó con las características adscriptas a las particularidades del comportamiento alimentario del propio niño: "aunque le dé de comer, es ella [por Cecilia] la que no quiere, yo trato, trato, pero tampoco puedo obligarla, hago lo que puedo" (Fernanda, 33 años con diagnóstico de desnutrición crónica). "[Manuel] es...como te puedo decir, mañoso, no es como los hermanos que te comen todo, él un poco hace lo que quiere" (Marina, 31 años, madre de un niño de 4 años con indicadores de desnutrición aguda). Así, si bien las madres se ocupan de los niños, éstos podían ser definidos en términos de características idiosincrásicas frente a las cuales sólo cabía la resignación.

Tanto la reafirmación del rol como la apelación a las particularidades del comportamiento de los niños, confluyeron en un efecto fundamental: imprimir entre la etiología del padecimiento definida por el sector salud y la perspectiva doméstica de su realidad somática cierta distancia irrevocable: en el primer caso, la afirmación del rol implicó un cuestionamiento de la perspectiva médica. Si las madres consideraban que se ocupaban de los niños y su alimentación, estos no podían estar "desnutridos". En los dos siguientes, el diagnóstico no era necesariamente cuestionado, pero la causa de la "malnutrición" se proyectaba fuera de las actividades vinculadas con el rol materno, para ubicarse en las condiciones estructurales o en el comportamiento indómito del niño.

Si bien algunos trabajos clásicos en sociología de la salud han destacado que la designación de determinado evento o comportamiento como patológico evita el juicio moral que pesa sobre quien lo padece ${ }^{25,26}$, la "desnutrición", como la mayoría de los padecimientos vinculados al comportamiento humano, es esquiva a esta tenden- cia. El diagnóstico es necesariamente un juicio moral, una distinción que jerarquiza la realidad somática de acuerdo a criterios valorativos sustentados en el saber biomédico. Pero además, en la medida en que el cuerpo diagnosticado y especialmente la etiología del padecimiento se compongan de elementos contextuales que exceden el terreno de lo biológico, estas dimensiones, aún si no son nombradas, se ven en alguna medida involucradas. No es que los profesionales juzguen a las madres o cuidadores por aquello que hacen o han hecho con sus hijos, sino que el diagnóstico y su etiología implican una jerarquización valorativa de la que necesariamente participan, con variadas interpretaciones y prácticas, tanto los profesionales como las madres y cuidadores.

La distancia establecida en el ámbito doméstico con la construcción clínica del cuerpo de los niños contribuyó a hacer de los espacios de intervención un campo conflictivo. Sus posibilidades de realización resultaron ajenas a la identificación de signos y síntomas que sus destinatarios calificaban como indeseables. En estas condiciones, el elemento central que posibilitó la existencia y eventual continuidad del dispositivo asistencial fue la caja de alimentos que los grupos percibían como beneficio adicional por ingresar al programa: “ $y$ si, tenés que ir porque si no, no te dan la caja, es cierto que a los chicos hay que hacerlos ver y todo, no te digo que no, pero para mí, lo fundamental es la caja." (Elsa, 31 años, madre de un niño de 4 con indicadores de desnutrición aguda); "yo por ahí no iría tanto viste, porque al final es un poco siempre los mismo. Si al chico lo veo mal, si tiene alguna enfermedad lo primero que hago es ir al centro de salud, pero después, si no fuera por la caja, no iría siempre como te hacen ir" (Cynthia de 35 años, madre de un niño de 4 con indicadores de desnutrición crónica).

Los complementos alimentarios, al tener como prerrequisito la concurrencia a las actividades asistenciales programadas por el sector salud, obligaban al sostenimiento de la visión clínica del cuerpo de los niños, al menos en las interacciones que estos tenían con sus profesionales. Esta aceptación instrumental no implicó una introyección de los criterios perceptivos y valorativos que constituían la mirada médica. Luego del diagnóstico, la alimentación infantil continuó sustentándose a partir del repertorio habitual de comidas que nutrían al conjunto de los integrantes de sus grupos domésticos. No se observaron preparaciones especialmente destinadas a los niños en general o a los "desnutridos" en particular. Esta falta de "adherencia”, era observada por 
los profesionales de la salud como una cualidad patologizable, adscripta generalmente a la psicología de sus integrantes en general y la de las madres en particular, o visualizada como uno de los efectos de la pobreza extrema en los espacios domésticos. Si estas dimensiones eran involucradas en el tratamiento lo tornaban aún más exigente en cuanto a la cantidad de profesionales que debían intervenir y los espacios institucionales que los niños y sus familias debían frecuentar. Las interconsultas con trabajadores sociales, psicólogos o psicopedagogos, extendían los dispositivos terapéuticos y los tiempos que los grupos domésticos debían dedicar al proceso de atención. Consecuentemente, la intención de abordar la creciente complejidad etiológica de los casos podía socavar la participación de los grupos en el tratamiento. En este contexto el programa adoptó el carácter de un conjunto diversificado de prácticas amenazadas por la indiferencia y la ruptura. La intencionalidad programada de sus profesionales continuó de esta manera lejos de los objetivos estipulados.

\section{Conclusiones}

Tanto el protagonismo asignado a los saberes médicos en la designación y el tratamiento de la desviación como el impacto de los dispositivos terapéuticos en la construcción de determinadas subjetividades, contribuyen a ubicar a los actores como sujetos pasivos de las prácticas y las representaciones que priman en las instituciones de salud. Sin embargo, el caso presentado nos permite destacar que la población lega puede elaborar cierta distancia con relación a la perspectiva profesional de su realidad somática. Si el buen paciente es aquel que se encuentra gobernado por un conjunto de normas perceptivas y morales propias del ámbito medico, los niños con "desnutrición" no resultaron ser buenos pacientes. $\mathrm{Ni}$ el contacto fluido con el sistema de salud, ni el hecho de que éste fuese considerado como el recurso primario en la atención de los padecimientos, afirmaron en los hogares a la "desnutrición" como un problema de salud que motivara una intervención específica e inmediata.

Este fenómeno se asentó en ciertas contradicciones centradas en las formas en que los profesionales de la salud y los destinatarios de sus acciones construyeron la realidad somática de los niños y sus contextos alimentarios. Desde el sector salud, la epistemología dominante fundamentó una diferencia absoluta en la percepción del cuerpo que, de acuerdo a los principios morales que regulan la identidad de sus profesionales, requería una acción inmediata. Más allá de los matices discursivos, el culturalismo etiológico proyectado sobre el padecimiento posibilitó la satisfacción de este imperativo moral, asentando la intervención de acuerdo con objetivos y recursos legítimos de la acción profesional: la promoción de hábitos alimentarios saludables mediante dispositivos educativos.

A pesar de su posición hegemonía, la construcción clínica sobre el cuerpo de los niños y sus contextos alimentarios no encontraron en los espacios domésticos un correlato sustentable. Frente al diagnóstico y la etiología postulados por el sector salud, madres y cuidadores realizaron un trabajo de restitución del orden perturbado a partir de dos pilares fundamentales: la continuidad contextual en la percepción corporal y la reafirmación de ciertos valores que componen el horizonte moral de la maternidad. Estos elementos, sustentados en la experiencia práctica de la supervivencia alimentaria, hicieron que el cuerpo de los niños se posicione como un centro de disputas entre los profesionales y los destinatarios de sus acciones. Si bien las prestaciones alimentarias involucradas en la implementación del programa asistencial permitieron la perpetuación de sus interacciones, el aura agonística del territorio en el que se producían marginó las respuestas y resultados que los profesionales esperaban como consecuencia de su intervención.

Este escenario revela dimensiones no siempre destacadas por quienes trabajan desde distintas perspectivas teóricas y metodológicas los contextos en los que se produce la medicalización. La complejidad de los procesos de salud, enfermedad y atención, invita a considerar sus distintas dimensiones no sólo desde el ángulo de la imposición, sino también de las múltiples formas de resistencia, transacción o identificación que componen la realidad social y sanitaria. La percepción y valoración del cuerpo, las patologías que se le adscriben, su inteligibilidad etiológica, las concepciones de riesgo y las eventuales prácticas de cuidado, son producto de un campo de heterogéneo de sentidos en el que participan, con disímiles capacidades de intervención, los diferentes conjuntos sociales. Estos elementos, introyectados como un precipitado de experiencias a partir de las cuales se construye el presente, componen las prácticas que en definitiva definen el complejo proceso de salud enfermedad y atención. Las singularidades de estas tensiones, los elementos que las componen y las múltiples formas en que se 
desarrollan en escenarios locales, permiten observar hasta que punto, tanto en los procesos de medicalización como en sus eventuales consecuencias en torno a la gobernabilidad, el sector salud constituye un actor ineludible, pero no un ordenador absoluto.

\section{Referencias}

1. Freidson E. Profession of medicine. Chicago: The University of Chicago Press; 1988.

2. Conrad P, Schneider J. Deviance and Medicalization. From Badness to sickness. Philadelphia: Temple University Press; 1992.

3. Menéndez E. Morir de alcohol. México: Ediciones de la Casa Chata; 1990.

4. Fassin D. Public health as a culture. The social construction of the childhood lead poisoning epidemic in France. Br Med Bull 2004; 69(1):167-177.

5. Herkovits D. Práxis profesional y realidad clínica: la construcción de la desnutrición infantil como objeto terapéutico en un centro de atención primaria en la ciudad de Buenos Aires. Cuadernos de Antropología Social 2007; (25):191-209.

6. Foucault M. Surveiller et punir. Naissance de la prison. Paris: Gallimard; 1975.

7. Foucault M. Histoire de la sexualité. Tome 1: La volonté de savoir. Paris: Gallimard; 1976.

8. Argentina. Ministerio de Salud y Ambiente de la Nación (MSAN). Manual metodológico de capacitación del equipo de salud en crecimiento y nutrición de madres y niños. Dirección de Salud Materno infantil. Buenos Aires: MSAN; 2003.

9. Geertz C. La descripción densa. In: Geertz C, organizador. La interpretación de las culturas. Barcelona: Gedisa; 2000. p. 19-40.

10. Hammersley M, Atkinson P. Etnografia. Barcelona: Paidos; 1994.

11. Patton M. Qualitative evaluation and research methods. Londres: Sage; 1990.

12. Miles MB, Huberman AM. Qualitative Data Analysis. An Expanded Sourcebook. Thousand Oaks: Sage; 1994.

13. Emerson R, Fretz R, Shaw L. Writing Ethnographic Fieldnotes. Chicago: The University of Chicago Press; 1995.

14. Souza Minayo MCS. El desafío del conocimiento: investigación cualitativa en salud. Buenos Aires: Lugar; 1997.

15. Strauss A. Qualitative Analysis for Social Scientist. Cambridge: Cambridge University Press; 1999.

16. Instituto Nacional de Estadísticas y Censos (INDEC). [site na Internet]. [acessado 2012 ago 15]. Disponibles en: http://www.indec.gov.ar.
17. Argentina. Buenos Aires. Programa de Vigilancia Nutricional del Gobierno de la ciudad de Buenos Aires. Prevención Asistencia y Rehabilitación Nutricional Materno Infanto Juvenil. Argentina: Gobierno de la Ciudad de Buenos Aires, Secretaría de Salud, Dirección Adjunta de Atención Primaria; 2003.

18. Bourdieu P. La distinction. Critique sociale du jugement. Paris: Les éditions de minuit; 1979.

19. Ramos S. Las relaciones de parentesco y ayuda mutua en los sectores populares urbanos: Un estudio de caso. Buenos Aires: Centro de Estudios de Estado y Sociedad (CEDES); 1984.

20. Aguirre P. Hiperinflación-estabilización en las estrategias domésticas de consumo de familias en situación de extrema pobreza. Cuadernos Médico Sociales 1991; (57):13-33.

21. Aguirre P. Estrategias de consumo: qué comen los argentinos que comen. Buenos Aires: Editorial Miño y Dávila; 2005.

22. Bourdieu P. Le sens pratique. Paris: Éditions de Minuit; 1980.

23. Bourdieu P. Sur la théorie de l'action. Paris: Le Seuil; 1994.

24. Herzlich C. Quelques aspects des représentations sociales de la santé et de la maladie. Revue Psychology Française 1964; (9):1-14.

25. Parsons T. The Social System. New York: Routledge; 1991.

26. Freidson E. Profession of medicine. Chicago: The University of Chicago Press; 1988.

Artigo apresentado em 12/08/2009

Aprovado em 24/02/2010

Versão final apresentada em 20/03/2010 